\title{
A New Method for Calculating Modeling Errors of Archimedes Hobs
}

\author{
Li Xue \\ Power system \\ Zhenjiang Campus of Army Military Transportation College \\ Zhenjian, Jiangsu, China \\ Lixue_cumt@sohu.com
}

\begin{abstract}
In this paper, Taylor series expansion is applied to calculate the modeling error of Archimedes gear hob. The solution of hob modeling error is changed from the original transcendental equation to the power series equation. Then it makes the calculation formula simple. Further clarification of the relationship between the parameters, easy to program calculation and computer optimization design. On this basis, a new formula for calculating Archimimedes helical tooth angle is proposed. It also has wide application value in Archimedes gear machining.
\end{abstract}

Keywords-Archimedes Hob, Involute Hob, Tooth Shape Error, Taylor Expansion

\section{INTRODUCTION}

In engineering mechanical transmission, most of the involute gears which have been widely used are machined by gear hobs. The meshing process of hob gears is essentially the meshing of staggered helical gears. According to the meshing principle of involute helical gears. The tooth profile of the end section of the gear should be involute. So the basic tooth profile of gear hob should be involute worm, the manufacturing and inspection of involute worm are difficult. As present, Archimedes worm is mostly used to replace the involute worm at home and abroad, so the forming error is produced[1-4].

So far, the modeling errors are calculated by complex transcendental equations(Abbreviated as transcendental equation method).The method is complicated in calculation, and the relationship between the forming error and the parameters is not clear, therefore, a new method of calculating the forming error of Archimedes hob by Taylor series expansion method is proposed in this paper, at the same time[5,6], a new method for calculating the tooth profile angle of Archimedes helical surface is put forward, which has wide practical value in practical application.

\section{THE BRIEF DESCRIPTION OF TRANSCENDENTAL EQUATION COMPUTING METHOD}

When hobbing gears, the forming errors of hobs are transmitted to gears along the direction perpendicular to the plane cutting the base cylinder of the involute worm, as show in Figure 1, make a tangent plane to the base cylinder of the involute worm, in which the cross section shape of the involute worm is a straight line I.The section shape of Archimedes worm is curve 2. I and II are tangent at the

\author{
Wang Guicheng \\ Silicon Lake College of Vocational Technology \\ School of Mechanical Engineering \\ Jiangsu University \\ Zhenjian, Jiangsu, China \\ 2026635795@qq.com
}

indexing circle. From any point on curve II (the radius of this point is $r_{\mathrm{y}}$ ) to line $\mathrm{I}$, we get $\Delta f_{n}$, that is the forming error of the point.

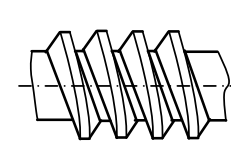

(a)

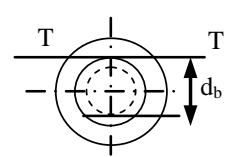

(b)

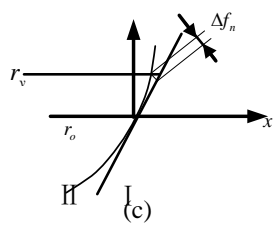

(c)

Fig.1. Forming error calculation chart

I: Section shape of involute worm in T-T-plane II: Section shape of Archimedes worm in T-T-plane

$$
\Delta f_{n}=\Delta f \cdot \cos \lambda_{b}=\Delta f \cdot \cos \lambda_{0} \cdot \cos \lambda_{a n}
$$

In Equation (1),

$\lambda_{b}$ - cylindrical helical angle of hob base;

$\lambda_{0}$-hob indexing cylindrical helix angle;

$\alpha_{n}$-hob normal tooth angle;

$\Delta f$ - axial forming error;

$\Delta f_{n}$-normal shaping error.

Because Archimedes worm manufactured has the same lead as the involute worm, so the helical surfaces of the two worms are tangent at the indexing circle. So on a cylindrical surface with any same radius, the observed $\Delta f$ is always equal in any axial section.

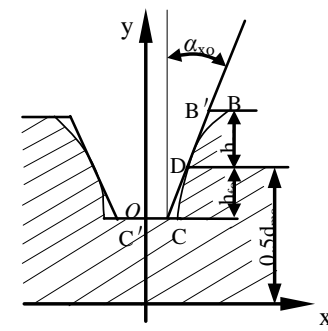

Fig.2. Axial Section Shape of Involute Worm and Archimedes Worm 
Fig. 2 shows the relationship between the cross-section shapes when Archimedes helicoid is used instead of involute helicoid in arbitrary cross-section. If coordinate axes ox and worm axes coincide with each other and oy axes coincide with the midline of the slot between teeth, the axial forming errors $\Delta f_{a}$ and $\Delta f_{f}$ measured by the top circle and the root circle of the hob in the horizontal direction are determined by Equation (2) and (3) respectively.

$$
\Delta f_{a}=\frac{m_{o} Z_{10}}{2 \cos \lambda_{o}}\left(i n v \alpha_{B}-i n v \alpha_{D}\right)-h_{a o} \tan \alpha_{x o}
$$

$$
\Delta f_{f}=h_{f o} \tan \alpha_{x o}-\frac{m_{o} Z_{10}}{2 \cos \lambda_{o}}\left(i n v \alpha_{D}-i n v \alpha_{C}\right)
$$

For the axial error between Archimedes helical surface and any point on cylinder $d_{x}$, Equation (4) is obtained by calculation and sorting out.

$$
\Delta f_{x}=\frac{m_{o} Z_{10}}{2 \cos \lambda_{o}}\left(i n v \alpha_{x}-i n v \alpha_{D}\right)-h_{x} \tan \alpha_{x o}
$$

\section{In Equation (4),}

$m_{o}$-normal modulus on indexing circle;

$\alpha_{B}, \alpha_{D}, \alpha_{C}$-pressure angle of Axis section of involute helicoid;

$\alpha_{x o}$-inclination of Archimedes helical busbar;

$Z_{10}$-number of hob heads;

$d_{x}$ - diameter of any point of hob;

$d_{m o}$-diameter of hob indexing cylinder;

Formula 4 is the general formulas for calculating the forming error of Archimedes hob, which can be seen from the formulas:

(1) When calculating with this formula, the calculation formula is complex and the amount of calculation is large.

(2) When the error is determined, the relationship between the parameters is not very clear.

(3) This formula is not conducive to programming and optimization design.

\section{APPLICATION OF TAYLOR EXPANSION METHOD}

In the design of gear hob, as long as the error can be controlled within a certain range, it can meet the actual needs of production, so the derivative values at point $d_{\text {mo }}$ are determined by Taylor expansion based on hob indexing cylinder in Equation (4). If the first four terms of the expansion formula are accurate enough for the practical application of hob design, the specific calculation is as follows.

$$
\begin{gathered}
\cos \alpha_{x}=\frac{d_{b}}{d_{x}} \\
\alpha_{x}=\cos ^{-1} \frac{d_{b}}{d_{x}}
\end{gathered}
$$

Therefore $\tan \alpha_{x}=\sqrt{\frac{d_{x}^{2}-d_{b}^{2}}{d_{b}^{2}}}$

So Equation (4) becomes:

$$
\tan \alpha_{x}=\sqrt{\frac{d_{x}^{2}-d_{b}^{2}}{d_{b}^{2}}}
$$

$$
\begin{aligned}
& \Delta f_{x}=\frac{m_{o} Z_{10}}{2 \cos \lambda_{o}}\left(\sqrt{\frac{d_{x}^{2}-d_{b}^{2}}{d_{b}^{2}}}-\cos ^{-1} \frac{d_{b}}{d_{x}}-i n v \alpha_{D}\right) \\
& -\frac{1}{2}\left(d_{x}-d_{m o}\right) \tan \alpha_{x o}
\end{aligned}
$$

Since Taylor's expansion is (Expansion at point $d_{m o}$ ):

$$
\begin{aligned}
& \Delta f_{x}=\Delta f_{x o}+\Delta f_{x o}^{\prime}\left(d_{x}-d_{m o}\right)+\frac{\Delta f_{x o}^{\prime \prime}}{2 !}\left(d_{x}-d_{m o}\right)^{2} \\
& +\frac{\Delta f_{x o}^{\prime \prime \prime}}{3 !}\left(d_{x}-d_{m o}\right)^{3}
\end{aligned}
$$

So the derivative of Equation (4) is calculated.

$$
\Delta f_{x o}=0
$$$$
\Delta f_{x o}^{\prime}=\frac{\tan \alpha_{n}}{2 \cos \lambda_{o}}-\frac{1}{2} \tan \alpha_{x o}
$$

$$
\Delta f_{x o}^{\prime \prime}=\frac{m_{o}^{2} Z_{10}^{2}}{2 \cos \lambda_{o} d_{m o}^{3} \tan \alpha_{n}}
$$

$$
\Delta f_{x o}^{\prime \prime \prime}=\frac{1}{\tan \alpha_{n} \cos \lambda_{o}} \cdot \frac{m_{o}^{2} \cdot Z_{10}^{2}}{2 d_{m o}^{3} \cdot d_{m o}}
$$

In order to estimate the error quantitatively,the tooth height $\mathrm{h}_{\mathrm{x}}{ }^{*}=\mathrm{h}_{\mathrm{xo}} / \mathrm{m}$ is introduced(the coefficient of top height of teeth is $\mathrm{h}_{\text {ao, }}^{*}$ the root height coefficient is $\mathrm{h}_{f_{0}}$ ). By substituting the above formulas into Taylor expansion,the normal forming error $\Delta \mathrm{f}_{\mathrm{xn}}$ at any point is obtained as Equation (5). 


$$
\begin{aligned}
& \Delta f_{x n}=h_{x}^{*} m_{o} \cos \alpha_{n}\left[\left(N-\tan \alpha_{x o}\right) \cos \lambda_{o}\right. \\
& \left.+A-2 A h_{x}^{*} m_{o} / d_{m o}\right]
\end{aligned}
$$

In formula:

$$
\begin{array}{r}
A=m_{o}^{3} Z_{10}^{2} h_{x}^{*} /\left(d_{m o}^{3} \tan \alpha\right) \\
N=\tan \alpha / \cos \lambda_{o}=\tan \alpha_{x o}
\end{array}
$$

Equation (5) shows that the value of $\triangle f_{x n}$ depends on $a_{x o}$. Because of the forming method,the involute helical surface and Archimedes helical surface only tangent at the indexing circle, but the error is not equal at the top circle and the root circle.But the error value can be minimized by choosing the appropriate $\mathrm{a}_{\mathrm{xo}}$ to make $\triangle \mathrm{f}_{\mathrm{a}}=\triangle \mathrm{f}_{\mathrm{t}}$.Therefore , if Equation (2) and Equation (3) are equal, Equation (6) can be obtained.

$$
\tan \alpha_{x o}=\frac{m_{o} Z_{10}\left(i n v \alpha_{B}-i n v \alpha_{C}\right)}{2\left(h_{a o}+h_{f o}\right) \cos \lambda_{o}}
$$

In fact, a similar relation can be obtained from Equation (5)

$$
\begin{aligned}
& \tan \alpha_{x o}=N+R\left\{\left(h_{a o}^{*}-h_{f o}^{*}\right)-Z_{10} m_{o}\right. \\
& \left.\left[\left(h_{a o}^{*}\right)^{2}-h_{a o}^{*} h_{f o}+\left(h_{f o}^{*}\right)^{2}\right] / d_{m o}\right\}
\end{aligned}
$$

In Equation (7),

$$
R=m_{o}^{2} Z_{10}^{2} /\left(d_{m o} \tan \alpha \cos \lambda_{o}\right)
$$

Assuming $h_{a o}^{*}=h_{f o}^{*}=1, Z_{10}=1$ (For standard hobs), Equation (7) becomes

$$
\tan \alpha_{x o}=N-2 \sin ^{3} \lambda_{o} \tan \lambda_{o} / \tan \alpha_{o}
$$

Equation (6) and Equation (7) are new formulas for calculating the tooth profile angle of the replace Archimedean helical surface. For the standard hob, the formula for calculating the tooth profile angle is Equation (7). According to Equation (7), the tooth shape angle $a_{x o}$ can be calculated in several seconds, which can fully meet the actual needs of production. After determining the tooth shape angle $\mathrm{a}_{\mathrm{xo}}$ of the hob according to Equation (7), the normal forming errors of the top and root of the hob are shown in Equation (8).

$$
\Delta f_{a n}=\Delta f_{f n}=K Q h_{a o}^{*} h_{f o}^{*}\left\lfloor 1+2 m_{o}\left(h_{f o}^{*}-h_{a o}^{*}\right) / d_{m o}\right\rfloor
$$

Among:

$$
K=\cos ^{2} \alpha / \sin \alpha ; Q=m_{o}^{4} Z_{10}^{2} / d_{m o}^{3}
$$

As can be seen from Equation (8).

(1) $\triangle f_{a n}\left(\triangle f_{f n}\right)$ decreased with the increase of $d_{m o}$ and $\alpha$;

(2) $\triangle f_{a n}\left(\Delta f_{f n}\right)$ increased with the increase of $\mathrm{m}_{\mathrm{o}}, \mathrm{Z}_{10}$ and $\lambda_{0}$;

(3) When $\lambda_{o}$ is a constant, the error is proportional to the modulus. Thus, Equaiton (8) reveals the relationship between errors and main parameters, and explains the influence degree of each parameter in them. Using this formula, errors can be quickly obtained and external diameter, head number and other related parameters can be selected.

In addition, Equation (8) is easy to be programmed and can be optimized by computer,thus reducing the workload of hob design and facilitating practical application.

\section{EXAMPLE OF CALCULATION}

In order to evaluate the accuracy of the calculation errors of the obtained formulas, the comparative calculations are made and shown in Table I using the data in Reference [2].

TABLE I COMPUTATIONAL ERROR COMPARISON

\begin{tabular}{c|c|c|c}
\hline \multicolumn{2}{c|}{ Hob parameter(mm) } & \multicolumn{2}{|c}{ Error( $\boldsymbol{\mu} \mathbf{~ m})$} \\
\hline $\mathrm{m}_{\mathrm{o}}$ & $\mathrm{d}_{\mathrm{mo}}$ & document 2 & Formula (8) \\
\hline 3 & 61.6 & 1.0 & 1.0 \\
5 & 76.2 & 4.0 & 4.1 \\
8 & 103.2 & 11.0 & 11.1 \\
14 & 142.4 & 41.0 & 41.2 \\
\hline
\end{tabular}

(1) Assuming that the parameters of the hob are: $Z_{10}=1, h_{f o}^{*}=1, \alpha=20^{\circ}$, the error values calculated according to formula 8 are shown in table I.

(2) Assuming that the parameters of the hob are: $m_{o}=12 \mathrm{~mm}, d_{m o}=14.67 \mathrm{~mm}, Z_{10}=1$, the error values calculated according to Equation (8) are shown in Table II.

From the calculation results in Table I and Table II, it can be seen that the forming error of hobs can be controlled within $3 \%$ by Taylor expansion method, which can fully meet the actual needs of production.

TABLE II CALCUALTION RESULTS

\begin{tabular}{c|c|c|c|c|c|c}
\hline & \multicolumn{4}{|c}{ A is the error value for the following values( $\mu$ m) } \\
\hline & \multicolumn{2}{|c|}{$1^{\circ}$} & \multicolumn{2}{c|}{$1^{\circ}$} & \multicolumn{2}{c}{$5^{\circ}$} \\
\hline$h_{x}^{*}(\mu \mathrm{m})$ & $\begin{array}{c}\text { Document } \\
2\end{array}$ & Formula 8 & $\begin{array}{c}\text { Document } \\
2\end{array}$ & Formula 8 & $\begin{array}{c}\text { Document } \\
2\end{array}$ & Formula 8 \\
\hline+1.0 & 20.5 & 19.9 & 14.4 & 14.3 & 11.0 & 10.8 \\
+0.5 & 5.6 & 5.5 & 3.7 & 3.9 & 2.9 & 2.9 \\
-0.5 & 6.5 & 6.5 & 4.3 & 4.4 & 3.5 & 3.5 \\
-1.0 & 28.8 & 27.9 & 20.4 & 20.1 & 15.4 & 15.3 \\
\hline
\end{tabular}




\section{CONCLUSION}

(1)In this paper, Equation (8) for calculating the forming error of Archimedes hob is derived by using Taylor expansion.it can reduce the difficultly of calculation and facilitate the programming of design.

(2) Equation (8) can intuitively explain the influence of each parameter on the error. Its advantage is that they have wide practicability.

(3)The new Equation (6) and (7) for calculating the tooth profile angle of Archimedes helical surface are proposed. The accuracy of the formulas can reach several seconds, which can be recommended for design calculation.

\section{ACKNOWLEDGEMENT}

This work was supported by the NSFC (№51075192), the National Science and Technology Major Project
(ZX201304009031) and Suzhou key laboratory of elevator safety technology.

\section{REFERENCES}

[1] Lu KaiJiang, Yang Feng. Principle Error Calculation of Machining Involute Tooth Profile with Archimedes Hob[J]. Journal of Baoji College of Arts and Sciences. 2002(12).

[2] Liu Dong Hui,Zhang Shu Seng. Discussion on the Theory and Design Method of Gear Hob Tooth Shaping[J]. Tool Engineering. 2001(2).

[3] Han Yu Ping,Wang YongMei. Forming Error and Correction of Archimedes Gear Hob[J]. Mechanical development. 2000(2).

[4] Yuan Zhe-Jun. Design of Gear Cutting Tool[M].Beijing: National Defense Industry Press,2014:152-158.

[5] Lu Zhong-Jian. Metal Cutting Principle and Cutting Tool [M].Beijing: Machinery Industry Press,2016:87-90.

[6] Tian Pei-Tang. Handbook for Design and Selection of Gear Cutting Toolsl [M].Beijing: National Defense Industry Press,2011:65-68. 